\title{
Corela
}

Cognition, représentation, langage

HS-4 | 2006

Le parcours

\section{WH- : Fin de parcours}

\section{Lionel Dufaye}

\section{OpenEdition}

Journals

Édition électronique

URL : http://journals.openedition.org/corela/1300

DOI : $10.4000 /$ corela. 1300

ISSN : 1638-573X

\section{Éditeur}

Cercle linguistique du Centre et de l'Ouest - CerLICO

\section{Référence électronique}

Lionel Dufaye, « WH- : Fin de parcours », Corela [En ligne], HS-4 | 2006, mis en ligne le 08 juin 2006, consulté le 19 avril 2019. URL : http://journals.openedition.org/corela/1300 ; DOI : 10.4000/ corela. 1300

Ce document a été généré automatiquement le 19 avril 2019

\section{(c) (i) (3) (2)}

Corela - cognition, représentation, langage est mis à disposition selon les termes de la licence Creative Commons Attribution - Pas d'Utilisation Commerciale - Partage dans les Mêmes Conditions 4.0 International. 


\title{
WH- : Fin de parcours
}

\author{
Lionel Dufaye
}

\section{Le parcours : explicitation d'une indétermination}

1 À la lecture des notes de séminaire 83-84 d'Antoine Culioli (Culioli A. 1985 dans la bibliographie), on constate que l'opération de parcours, quelle que soit sa souscatégorisation (lisse, rugueux, avec sortie, avec totalisation, etc.), est essentiellement présentée en opposition à d'autres types d'opération de détermination.

2 Au niveau des déterminations qui affectent la relation prédicative, Culioli parle de parcours (lisse ou rugueux) par opposition à de la construction d'occurrences spécifiques dans des exemples comme :

The early bird catches the worm. (parcours lisse)

Il est arrive que ... (parcours rugueux)

3 À un autre endroit, le parcours est également assimilé à de la construction de classe, par contraste avec de l'occurrence particulière. Il s'agit de décrire des exemples comme

les chiens sont des mammifères ou

tout chien est un mammifère,

que Culioli commente de la manière suivante :

"J'ai bien une opération de parcours dans la mesure où je me construis une classe

d'occurrences abstraites de <être chien> i.e. ce qui est un chien typique. » (Culioli

$1985: 72$ )

4 Les questions dites «fermées" (yes-no questions) sont également traitées comme des formes de parcours par opposition à de l'assertion. Les yes-no questions sont des opérations modales qui construisent une prise en compte orientée des différentes zones du domaine notionnel (IE) $\rightarrow \mathrm{I} \rightarrow \mathrm{E}(\rightarrow \mathrm{IE})^{1}$.

5 À un niveau plus argumental, on a le problème des questions dites « ouvertes » (de type WH-), où le parcours s'interprète en opposition à une assignation de valeur référentielle pour un argument donné. Le cas des pronoms interrogatifs est également relié à des phénomènes de modalité, où le parcours est commenté en opposition avec l'opération de « centrage»: 
Où peut-il bien être?

* Où peut-il fort bien être?

6 Culioli explique ainsi que « fort » construit un centrage par rapport au centre attracteur qui rend le parcours incompatible.

Enfin, dans un article de 1987, Culioli (1990 dans la bibliographie) présente explicitement le parcours en contraste avec l'extraction et le fléchage :

«Le parcours à la différence de l'extraction et du fléchage, fait que l'on ne peut (ou

veut) distinguer aucune occurrence dans le domaine. »(Culioli $1990: 121$ )

7 De sorte qu'au niveau du groupe nominal, par exemple, on en vient ainsi à se représenter un système qui se caractérise par différents degrés de détermination :

- Absence de détermination

- Parcours

- Extraction

- Fléchage.

La première remarque que l'on peut faire, à la lecture des textes de Culioli $(1985,1990$ a et b, 1999), est que l'on se forme une représentation de ce qu'est l'opération de parcours à partir de sa confrontation avec d'autres opérations. Dans tous les cas, on comprend que l'on a parcours lorsqu'on a absence de stabilisation d'une valeur: il peut s'agir de stabilisation d'une valeur modale comme l'assertion, d'une valeur argumentale comme avec les marqueurs en Wh-, ou encore d'une valeur occurrencielle comme dans le cas du spécifique. Dès lors qu'il y a instabilité, il y a parcours, ce qui amène à définir le parcours en négatif puisqu'on l'appréhende essentiellement en regard de ce qu'il ne fait pas.

Il semble néanmoins que l'on trouve une forme de définition pour le parcours dans la citation suivante (c'est moi qui souligne) :

«La notion de parcours est liée à la construction d'une classe d'occurrences abstraites d'une notion. On parlera de parcours de la classe K. Vous la parcourez sans que vous vouliez ou puissiez vous arrêter à une valeur distinguée parmi les autres valeurs. Dans certains cas, vous ne pouvez pas et vous aurez éventuellement recours à autrui; dans d'autres, vous ne voulez pas, et vous allez avoir une modalisation sans recours à autrui. »(Culioli 1985 : 70)

On voit que la $1^{\text {ère }}$ phrase nous informe sur le fait que le parcours est une opération dont l'argument doit être "une classe d'occurrences abstraites ». Avec la $3^{\text {ème }}$ phrase, on comprend que le principe de cette opération est de prendre en compte ces occurrences abstraites sans (vouloir ou pouvoir) en distinguer aucune. Enfin, l'idée selon laquelle « on ne veut ou peut s'arrêter à une valeur distinguée » suggère une opération dynamique, qui appréhenderait les occurrences séquentiellement plutôt que simultanément. L'étymologie véhiculait déjà ce sens puisque per + currere signifie d'abord "courir à travers ». Le Robert et Le Robert historique de la langue française donnent ainsi les acceptions suivantes: 16e s., "traverser, visiter dans toute son étendu ", "aller dans toutes les parties d'un lieu », « regarder successivement les éléments d'un ensemble (pour avoir une vue générale) »; 17e s., « aller d'un bout à l'autre »; 18e s., « accomplir un trajet déterminé ».

10 Cette opération, que l'on pourrait qualifier de " cinétique ", fait du parcours un mode de détermination particulier, dans la mesure où, en raison du manque de stabilisation qu'il suppose, il s'agirait plutôt d'une forme d'indétermination. En effet, le Robert historique nous rappelle que « déterminer » signifie d'abord " marquer les limites, borner, limiter », et au figuré, « arrêter, régler, fixer ». Dans la mesure où Culioli définit le parcours comme 
une incapacité ou un refus de "s'arrêter à une valeur distinguée ", l'idée d'une «opération d'indétermination" ne semble pas inappropriée pour qualifier le parcours. Cette notion intervient d'ailleurs dans les analyses de Gresset 1984 (p. 262), Rossignol 1984 (p. 262), Culioli 1990 (p. 166).

11 À ce stade, le parcours s'oppose à deux formes de détermination. D'un côté, il est une forme d'indétermination dans la mesure où il se distingue d'opérations comme l'extraction et le fléchage, qui construisent de la délimitation d'occurrences. D'un autre côté, le parcours est une forme de détermination, à la différence des phénomènes d'adétermination comme l'absence de marqueur dans le cas $\mathrm{du} \mathrm{GN}$ ou encore l'aoriste dans le cas de l'aspect. En effet, à la différence d'une a-détermination, on associe toujours le parcours à des marqueurs comme wh-, any, ever, la forme interrogative, les constructions concessives, etc. (le but de cet article est de savoir si cette association est justifiée). Dans d'autres approches théoriques, l'assimilation entre l'opération de parcours et des marqueurs particuliers est explicitée dans la désignation métalinguistique. Ainsi, Jayez \& Tovena (2003) parlent de Free Choice Items (sur le modèle des Negative Polarity Items), donnant ainsi l'idée d'une classe définie de marqueurs identifiables.

12 Ainsi, envisagé au sein du système de détermination, le "parcours» a un statut ambivalent: il construit bien une détermination, mais tout en explicitant de l'indétermination. Pour cette raison, sans doute peut-on concevoir les marqueurs de parcours comme les traces d'une indétermination construite.

13 Evidemment, l'indétermination n'est un critère suffisant ni pour définir ni pour identifier le parcours. Pourtant, lorsqu'on observe les contextes dans lesquels le concept apparaît, force est de reconnaitre que l'étiquette métalinguistique de "parcours » - avec son sens cinétique et la définition qui lui fait écho - se présente avant tout comme une métaphore intuitive de l'absence d'individuation. Ce qui pose le problème du recours à une métalangue issue du langage courant, avec les risques d'interférences entre le sens ordinaire et le sens technique. Après tout, on aurait pu envisager la mise en place d'un système de détermination avec une métalangue non lexicalisée et néanmoins pourvue de définitions ; ainsi, sur le modèle des modalités, on aurait pu imaginer une détermination de type 1, de type 2, etc. Dès lors que l'on opte pour une terminologie "figurée », il faut gérer la relation incontournable entre sens courant et le sens technique. Pour prendre l'exemple de la «quantité (Qnt) », on doit rappeler en permanence que "quantité » n'a pas le sens numérique qu'il a dans la langue quotidienne. Et cependant, l'étiquette " quantité » n'a pas non plus été choisie au hasard; de sorte que, aussi paradoxal que cela soit, les expressions métalinguistiques les plus "parlantes " nécessitent tout autant, sinon plus que les expressions neutres, l'appui d'une définition.

14 À côté de l'étiquetage de l'opération, on a le problème de son association à certains types de marqueurs. Ainsi, à mesure que les années passent de plus en plus de monde prend le train de la TOE en marche, héritant ainsi d'une réflexion théorique que l'on va avoir tendance à considérer comme acquise. Le risque est alors de voir s'installer des identifications implicites telles que «wh- / any / ever(y) ... sont des marqueurs de parcours ", qui peuvent mener à un renversement de situation, puisque le marqueur dont on cherche à rendre compte devient ce par quoi on accède au concept.

15 La question est de savoir s'il existe des critères formels pour déterminer si un marqueur donné est un opérateur de parcours (c'est-à-dire un marqueur qui se conformerait à la définition de Culioli citée plus haut)? En revanche, s'il s'agit d'une évaluation essentiellement intuitive, comment peut-on être sûr que l'on ne projette pas sur le 
marqueur en question ce qui relève en fait d'autres opérations contextuelles? Ainsi, lorsqu'on reconnait un parcours « lisse », un parcours « rugueux », un parcours " avec (ou sans) sortie », un parcours " avec totalisation », etc. (pour ne rien dire de l'énigmatique parcours « moiré »), comment distinguer ce qui relève du parcours lui-même des valeurs induites par le contexte?

\section{S'agit-il BIEN d'un marqueur de parcours?}

Pour prendre un exemple, dans Culioli 1985 on trouve l'idée que BIEN peut être soit un opérateur de parcours, soit un opérateur d'identification :

«BIEN peut être soit le marqueur d'une identification soit le marqueur d'une opération de parcours ; cf. : Tu finiras bien par... » (Culioli 1985 : 99)

Dans le contexte contemporain, où l'on est en recherche permanente de formes schématiques $^{2}$, on peut trouver surprenant d'associer ainsi deux opérations de détermination a priori contradictoires à un même marqueur. Or, à la relecture de l'analyse de BIEN (1985), on ne peut s'empêcher de s'interroger sur l'influence du contexte sur l'analyse du marqueur. Par exemple, partant de

*où peut-il fort bien être?

Culioli formule le commentaire suivant :

«Une interrogation est un parcours, ici sur la classe des occurrences dont 'où' est l'image ; 'bien' est le marqueur de cette opération de parcours : c'est compatible et c'est nécessairement situé puisque dans l'interrogation, vous avez relation construite par rapport à l'autre énonciateur. Si vous aviez introduit 'fort', vous auriez centré, or vous ne pouvez à la fois parcourir et centrer. » (Culioli 1985 : 100)

Il est dit à la fois qu' " une interrogation est un parcours » et que « 'bien' est le marqueur de cette opération de parcours ", de sorte qu'on peut se demander si l'on a affaire à un cumul de parcours, ou si c'est l'analyse d'un marqueur qui déborde en fait sur l'autre. À un autre endroit, BIEN (de même que POUVOIR) n'est pas présenté comme un parcours, mais comme « compatible » avec lui :

«Avec l'interrogation, on a un parcours sur toutes les valeurs possibles. [...]

« Pouvoir » est compatible avec ce parcours, « bien » l'est aussi. » (Culioli 1985 : 55)

Un peu plus loin, la distinction entre l'identification et le parcours de BIEN est accompagnée de quelques illustrations :

« Avec le marqueur BIEN il faut au moins deux occurrences. On peut avoir :

identification ex. Il est bien arrivé.

parcours ex.Où peut-il bien être?

parcours avec sortie ex. Tu finiras bien par..., Il arrivera bien... »

(Culioli 1985 : 56)

On constate que BIEN n'est associé à du parcours que s'il y construction d'un hiatus dans le contexte. Ainsi, finir / arriver + le futur construisent ici une distance entre Sito et une borne de changement d'état, ce que note explicitement Culioli :

«Si je travaille sur du futur, c'est une forme qui porte sur du non-certain. Si l'on emploie BIEN, il va immédiatement vous venir à l'esprit 'en fin de compte', or 'finir' est par excellence le passage d'une zone à une autre : le franchissement d'une frontière. » (Culioli 1985 : 56)

On peut en fait considérer avec Culioli que «bien marque le renforcement de ce chemin » $(1990,158)$ sans faire intervenir le parcours, ce qui reste d'ailleurs en accord avec la 
définition qu'il avance au début du même article, et qui ne mentionne aucunement le parcours :

«Bien marque une opération complexe par laquelle (a) on construit un domaine notionnel (intérieur centré, frontière, extérieur), (b) on situe un terme (une occurrence) par rapport à un autre terme (une autre occurrence) dans le domaine. » (Culioli 1990 : 157)

La non-pertinence du parcours est également vraie dans le cas de l'interrogative, qui se conforme à l'opération de parcours avec ou sans la présence de BIEN (voire avec ou sans la présence de POUVOIR):

S'il vit, où peut-il (bien) être ?3

On retrouve l'idée du renforcement d'un hiatus deux représentations modales, ce qui autorise par ailleurs la cooccurrence d'un marqueur disjonctif comme MAIS :

Mais où peut-il bien être ? Je ne le vois pas.

En d'autres termes, la présence d'une opération de parcours dans le contexte immédiat est compatible avec bien, qui «marque ce jeu d'entrée et de sortie, qui établit une relation de zone à zone » $(1990,160)$. Cependant, pour rendre compte des phénomènes en question, il n'est pas nécessaire de considérer BIEN comme un opérateur de parcours dans la mesure où cette opération est prise en charge pas d'autres marqueurs.

\section{Wh- Fin de parcours}

De manière comparable, Culioli (1985) propose une analyse de QU- (qui est assimilé formellement à $\mathrm{WH}^{-4}$ ), qui est mise en relation avec des opérations de parcours. À partir de là, on va voir s'installer une équivalence $\mathrm{WH}^{-} / \mathrm{QU}-=$ parcours, et ce, en dépit d'analyses concurrentes proposées à la même époque, comme le rappel Gournay $2005^{5}$ :

«En 1983, Danon-Boileau dans un article sur les relatifs ne mentionne pas le terme de parcours, et, en 1977, Chevillet lui décrit WH- comme un opérateur d'extraction. En tout cas après 1984, tous les articles sur les marqueurs en WH- que j'ai consultés, reprennent en chœur l'équation $\mathrm{WH}^{-}=$Parcours sans que cette équation soit justifiée ou discutée (même si parfois on trouve des références aux écrits de Culioli sur QUE). » (Gournay $2005: 61$ )

Or dans les notes du séminaire 1983-1984, on voit que QU- n'est pas, du moins dans un premier temps présenté comme du parcours. Culioli part de l'exemple

Est-ce qu'il est venu?

qu'il commente ainsi (c'est moi qui souligne) :

«Je pose 'il est venu' et j'effectue une opération de reprise par rapport à laquelle je fais comme si je la vidais de tout l'appareil énonciatif - c'est la reprise de quelque chose qui n'est plus une assertion mais qui a toutes les propriétés d'une assertion. C'est ce qui apparait avec: 'qu'il est venu'; ensuite je pose un parcours sur la possibilité de repérer par rapport à tout le système de coordonnées énonciatives: 'est localisable par rapport à', 'est identifiable à'. En français, cela renvoie à 'c'est le cas' ou 'ce n'est pas le cas'. » (Culioli1985:72)

Ainsi présenté, le parcours, dont les arguments sont les pôles du plan de validation (être le cas/ne pas être le cas), n'apparaît qu'après l'opération dont QUE est la trace. Le parcours ici encore est une opération construite dans le contexte immédiat ('est-ce'), en interaction avec QUE. Dans son article sur BIEN ([1987] 1990, pp. 157-168), Culioli décrit QUE comme la trace d'un paradigme de valeur sur lequel va s'appliquer le parcours : 
«Si l'on analyse une interrogation telle que Que peut-il bien faire?, on voit que Que est le représentant (l'image) du domaine des valeurs possibles et imaginables que l'on peut assigner à la place vide dans il fait ( ). Que permet de représenter une classe d'occurrences parmi lesquelles on ne peut pas (ou éventuellement, on ne veut pas) en distinguer une, qui permettrait la validation de la relation prédicative non saturée. » (Culioli 1990 : 164)

\section{"l'image » d'une classe
la forme interrogative :}

« Une interrogation est un parcours, ici sur la classe des occurrences dont « où » est l'image. »(Culioli 1985 : 100)

Pourtant, on constate l'opération de parcours va finalement être attribuée à WH-, comme le confirment les citations suivantes (pour ne prendre que quelques exemples):

"Which 'interrogatif' et WHICH 'relatif' ont, selon nous, la même valeur mais ils diffèrent dans la mesure où l'indétermination de parcours contenue dans WHICH persiste jusqu'à la réponse dans le cas de WHICH interrogatif [...] tandis que cette indétermination est levée par un renvoi à la situation d'énonciation dans le cas de WHICH relatif. » (Gresset, 1984, p. 262)

«On distingue de façon classique THAT et WHICH par le fait que le premier a une valeur fondamentale de type déictique alors que le second marque essentiellement une opération de parcours. » (Flintham 1993 : 172)

«Les QU- sont des « indéfinis (plus précisément des opérateurs de parcours). [...] cette opération de parcours originelle sous-tend la totalité de leurs emplois, même des emplois a priori très éloignés, comme celui du relatif. » (Le Goffic 1994 : 31)

«La forme en WH- est la trace d'un parcours des valeurs susceptibles d'instancier la place vide. » (Khalifa 1999 : 193)

«If, comme whether et les autres mots en wh-, marque une opération de parcours. » (Chuquet \& Hanote 2004 : 57)

Et dans la logique d'une sémantique qui considère qu'à chaque marqueur correspond une forme schématique (un invariant formel), WH- et QU- se voient qualifiés d'opérateurs de parcours au-delà de leurs emplois interrogatifs. On comprend par là même le point de vue de Gournay 2005 lorsqu'elle souligne « l'inadéquation qu'il y a à parler de 'parcours' pour WH- que ce soit dans un contexte interrogatif ou dans un autre $» .^{6}$

Comparons l'utilisation de WH- dans des contextes interrogatifs et relatifs proches en empruntant quelques exemples à $\mathrm{McCawley}^{7}$ (1988, pp. 431-432) :

I'll ask what he's selling. (interrogative imbriquée)

I'll buy what he's selling. (relative nominale)

I'll buy wether / if ...

31 L'interprétation en termes d'interrogative imbriquée ou de relative nominale est conditionnée par le sémantisme du verbe de la principale. Ainsi, des verbes comme buy, dont on remarque qu'ils sont incompatibles avec des complémentations impliquant une bifurcation, bloquent toute lecture interrogative ( ${ }^{*}{ }^{\prime} l l$ buy if / whether ...). En fait, la possibilité d'avoir une interrogative imbriquée repose sur au moins deux critères. Le premier critère est que le verbe de la principale accepte un complément propositionnel, alors que la relative peut-être analysée comme ayant un noyau nominal. C'est ce que montrent les tests d'accord cités par McCawley (ibid.) :

[What books [he's written]] haven't / *hasn't been sold yet.

[What books he's written] hasn't / *haven't been established. 
De même, on observe l'impossibilité de faire «monter » la préposition dans le cas de la relative nominale :

I'll ask what he was pointing at.

I'll ask at what he was pointing.

I'll buy what he was pointing at.

*'ll buy at what he was pointing.

Puisque ask accepte un complément propositionnel, on peut concevoir, selon les principes génératifs, un mouvement en WH- (avec ou sans pied piping) en position de spécifieur du $\mathrm{CP}$. En revanche, un verbe comme buy sous-catégorise un complément direct non propositionnel (nominal en l'occurrence), de sorte que WHAT peut être analysé comme un pronom dans les deux derniers exemples. Dans ces conditions, pas de pied piping puisque WHAT reste in situ, ce que l'on pourrait représenter un peu maladroitement de la manière suivante :

I'll ask [cp at what he was pointing [po at what ]po ]co

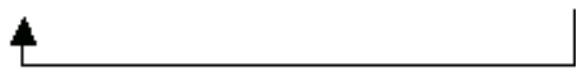

\section{I'll buy [no what [co $\varnothing$ he was pointing [po at $\varnothing$ jop ]co ]no}

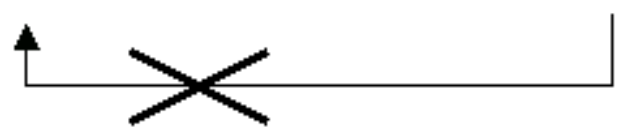

Le deuxième critère, qui est sémantique, est corrélé au premier puisque les interrogatives sont introduites par des prédicats que l'on qualifie parfois « d'attitude propositionnelle ${ }^{9}$ " (leur complément doit donc bien être une proposition). Dans le cas des interrogatives imbriquées, on a des prédicats qui ont pour propriété commune de suspendre toute prise en charge assertive (soit en raison de leurs propriétés lexicales - wonder, ask, be unclear, etc. -, soit par interaction entre les propriétés lexicales et des opérations périphériques know / tell... + modalité dés-assertive). Dans tous les cas, un paradigme de la proposition a une valeur non distinguée, ce qui implique que la validation de la proposition ne sera pas stabilisée. C'est par conséquent au niveau du prédicat que se met en place l'opération de parcours.

Le rôle des marqueurs en WH- est de spécifier l'argument de ce parcours. Pour ce faire, on a d'un côté WH- lui-même, qui est la trace d'un paradigme qui n'a pas été saturé (ce que Culioli note par des parenthèses vides), et d'un autre côté, on a le morphème suffixal, qui va déterminer la sémantique de ce paradigme :

avec wh-ether il s'agit des polarités de validation de la relation prédicative $\left(\mathrm{I} / \mathrm{E}^{10}\right)$; avec les autres marqueurs en WH-, le suffixe peut soit définir le sémantisme du syntagmes (wh-o, wh-ere, wh-en, etc.), soit définir un type de Qlt (Qlt non différentiel pour wh-at ; Qlt différentiel pour wh-ich).

Prenons un dernier exemple pour montrer que WH- n'est pas un opérateur de parcours, mais le site du parcours :

*I'll ask whatever he's selling.

I'll buy whatever he's selling.

I asked what ever we had done to deserve that.

Ici encore, on distingue une interrogative indirecte et une relative nominale. Or, quel est le problème? On a d'un côté un parcours posé par le sémantisme de ask, et d'un autre 
côté on a ever, dont le fonctionnement se conforme ici totalement à la définition du parcours (i.e. renvoi à une classe d'occurrences sans possibilité ou volonté de distinguer une valeur). Comment expliquer l'incompatibilité de ever avec l'interrogative, alors même qu'il est parfaitement acceptable avec la relative nominale?

Mon explication est que WH- (what ici) ne peut pas être le lieu de deux parcours différents. Autrement dit, si ever est possible avec les relatives libres c'est précisément parce qu'elles ne supposent aucun parcours ; il n'y a donc pas interférence. En revanche, dans le cas des interrogatives, WH- est déjà la cible d'un parcours. Or, ce parcours se caractérise par l'aboutissement d'une éventuelle assignation de valeur qualitative. Alors que ever ne construit pas de parcours avec sortie, d'où, semble-t-il, une incompatibilité de cumuler deux parcours différents pour un même argument. Plus d'incompatibilité en revanche dans le dernier cas puisque EVER, qui se désolidarise du marqueur en $\mathrm{WH}^{-}$, porte sur un paradigme modal distinct. Ainsi, au mieux, la présence de WH- nous autorise à soupçonner une opération de parcours, elle n'en constitue en rien une preuve. De même que le parcours n'implique pas WH-, WH- n'implique pas le parcours.

\section{BIBLIOGRAPHIE}

Chuquet, H. \& Hanote, S. (2004) Who's Speaking, Please? Le discours rapporté, Gap : Ophrys.

Culioli, A. (1985) Notes du séminaire de D.E.A. 1983-1984, éditées par le Département de Recherches Linguistiques : Université Paris VII.

Culioli, A. (1990) Pour une linguistique de l'énonciation, Tome I, Collection l'Homme Dans la Langue, Paris : Ophrys.

Culioli, A. (1999a) Pour une linguistique de l'énonciation, Tome II, Collection l'Homme Dans la Langue, Paris : Ophrys.

Culioli, A. (1999b) Pour une linguistique de l'énonciation, Tome III, Collection l'Homme Dans la Langue, Paris : Ophrys.

Danon-Boileau, L. (1983) «This, That, Which, What et la construction de références ", in Méthodes en linguistique anglaise, CIEREC XXXIX, Université de Saint-Etienne.

Flintham, R. (1993), « Les relatives en WHICH et THAT dans Scientific American », in Opérations énonciatives et interprétation de l'énoncé, pp. 171-179, Gap : Ophrys.

Gournay, L. (2005) « (Entre autres choses) pourquoi les marqueurs en WH- ne sont finalement pas des opérateurs de parcours », Parcours linguistiques, CIEREC travaux 122, Saint-Etienne :

Publication de l'Université de Saint-Etienne.

Gresset, S. (1984) « ТНAT/WHICH marqueurs de relatives en anglais contemporain », in Cahiers de recherche en grammaire anglaise, tome II, p. 202-272, Gap : Ophrys.

Khalifa, J.-C. (1999), La syntaxe aux concours, CAPES/Agrégation, théorie et pratique de l'énoncé complexe, Armand Colin, Paris. 
Lapaire, J.-R. \& Rotgé, W. (1991), Linguistique et grammaire de l'anglais, Toulouse : Presses universitaires du Mirail.

Le Goffic, P. (1994), « Indéfinis, interrogatifs, relatifs (termes en QU-) : parcours avec ou sans issue ", Faits de Langues, $n^{\circ} 4$, p. 31-40, PUF.

McCawley, J. (1988), The syntactic Phenomena of English I \& II, Chicago: UCP.

Rossignol A. (1984), «Ordre syntaxique, modalité et opérations de détermination », Cahiers CHARLES V n ${ }^{\circ}$, p. 111-126, Gap : Ophrys.

\section{NOTES}

1. IE se lit « extérieur renforcé » (qui correspond au plan de représentation), I se lit « Intérieur », et E se lit « Extérieur» (ces 2 zones constituent le plan de validation).

2. Les «formes schématiques» sont des représentations invariantes formelles.

3. L'énoncé d'origine est construit sans bien : Jules Verne, L'Ile mystérieuse, chap. 5.

4. "Quand j'ai ( ) je vais nécessairement avoir en français ce qu'on a appelé "qu-" ou en anglais "wh-".»

5. L'article étant en cours de publication, la pagination n'est pas connue.

6. L'article étant en cours de publication, la pagination n'est pas connue.

7. Exemples qu'il emprunte lui-même à : Bresnan, J. \& Grimshaw J. (1978), "The Syntax of Free Relatives in English", Linguistics Inquiry 9, pp. 331-391.

8. i.e. avec ou sans montée conjointe de la préposition.

9. Voir Benveniste, E. ([1958] 1966), « De la subjectivité dans la langue », Problèmes de linguistique générale 1, Tel Gallimard, pp. 258-276.

10. Dans le cas de whether $p$ or $q$ (or ...), on travaille également sur I / E, mais ce paradigme s'applique à des relations prédicatives mutuellement exclusives puisque renvoyant au même cadre de référence : whether $\mid \Leftrightarrow \mathrm{E}: \lambda 1$, or $\mid \Leftrightarrow \mathrm{E}: \lambda 2$, or $\ldots$

\section{RÉSUMÉS}

Un certain nombre de marqueurs ont été plus ou moins catégorisée comme des opérateurs de parcours : ever, any, l'interrogation, ... Cet article tente de montrer que pour certains d'entre eux - en l'occurrence BIEN en français et WH- en anglais - cette association doit être remise en cause dans la mesure où l'opération de parcours qui leur a été attribuée est en fait prise en charge par d'autres opérations dans le contexte. Pour défendre cette argumentation, nous relirons les analyses avancées dans les travaux d'Antoine Culioli, ce qui nous permettra également de préciser le concept de parcours.

A number of linguistic markers have been more or less identified as "scanning" operators: ever, any, interrogation ... In this article, I argue that for some of them - namely the French adverb BIEN and the English morpheme wH- - this association in inappropriate and should be questioned. I will actually try to show that the scanning operation that they purportedly represent is actually 
attributable to other contextual markers. This demonstration will be based on Antoine Culioli's analyses, which should help us put forward a more precise definition of the scanning operation.

\section{INDEX}

Mots-clés : bien, indétermination, étymologie (de « parcours »), ever, extraction, fléchage, interrogation, interrogative imbriquée, métalangue (métalinguistique), question fermée, question ouverte, relative nominale (relative libre), wh-

Keywords : bien, embedded interogation, etymology (of scanning), ever, extraction, free relative, Interrogation, Yes/No question, indetermination, metalanguage (metalinguistics), pinpointing, wh-, wh- question

\section{AUTEUR}

\section{LIONEL DUFAYE}

UFR Charles V, Paris 7 